\title{
Disentangling Bulk and Interface Phenomena in a Molecularly Doped Polymer Semiconductor
}

\author{
Dominique Lungwitz, Thorsten Schultz, Claudia E. Tait, Jan Behrends, Swagat K. Mohapatra, \\ Stephen Barlow, Seth R. Marder, Andreas Opitz, and Norbert Koch*
}

Dedicated to Professor Karl Leo on the occasion of his 60th birthday

Doping the electron-transport polymer poly $\left\{\left[N, N^{\prime}\right.\right.$-bis (2-octyldodecyl) naphthalene-1,4,5,8-bis(dicarboximide)-2,6-diyl]-alt-5,5'-(2,2'-bithiophene)\} [P(NDI2OD- $\left.\left.\mathrm{T}_{2}\right)\right]$ with the bulky, strongly reducing metallocene $1,2,3,4,1^{\prime}, 2^{\prime}, 3^{\prime}, 4^{\prime}$-octaphenylrhodocene (OPR) leads to an increased bulk conductivity and a decreased contact resistance. While the former arises from low-level $n$-doping of the intrinsic polymer and increased carrier mobility due to trap-filling, the latter arises from a pronounced accumulation of dopant molecules at an indium tin oxide (ITO) substrate. Electron transfer from OPR to ITO leads to a work function reduction, which pins the Fermi level at the P(NDI2OD-T 2 ) conduction band and thus minimizes the electron injection barrier and the contact resistance. The results demonstrate that disentangling the effects of electrode modification by the dopant and bulk doping is essential to comprehensively understand doped organic semiconductors.

\section{Introduction}

Molecular electrical doping is a powerful approach to control the charge carrier density and Fermi level position in organic semiconductors. By adding strong molecular electron acceptors (p-type doping) or donors (n-type doping), the density of positive or negative charge carriers, respectively, can be increased significantly. Precise control of the electronic properties of organic semiconductors is essential to enhance the performance of electronic devices such as organic light-emitting diodes, organic photovoltaic cells, and organic field-effect transistors. ${ }^{[1-4]}$ To date, efficient $\mathrm{n}$-type doping of organic semiconductors remains challenging. While a variety of stable molecular p-dopants has been successfully deployed in electronic

D. Lungwitz, Dr. T. Schultz, Dr. A. Opitz, Prof. N. Koch

Institut für Physik and IRIS Adlershof

Humboldt-Universität zu Berlin

D-12489 Berlin, Germany

E-mail: norbert.koch@physik.hu-berlin.de

Dr. T. Schultz, Prof. N. Koch

Helmholtz-Zentrum Berlin für Materialien und Energie $\mathrm{GmbH}$

D-12489 Berlin, Germany

Dr. C. E. Tait, Prof. J. Behrends

Berlin Joint EPR Lab

Fachbereich Physik

Freie Universität Berlin

D-14195 Berlin, Germany

Dr. S. K. Mohapatra, Dr. S. Barlow, Prof. S. R. Marder

School of Chemistry and Biochemistry and Center for

Organic Photonics and Electronics

Georgia Institute of Technology

Atlanta, GA 30332-0400, USA

Dr. S. K. Mohapatra

Department of Industrial and Engineering Chemistry

Institute of Chemical Technology-Indian Oil Odisha Campus

IIT Kharagpur Extension Center

Bhubaneswar, Odisha 751013, India

The ORCID identification number(s) for the author(s) of this article can be found under https://doi.org/10.1002/adom.202002039.

(C) 2021 The Authors. Advanced Optical Materials published by Wiley-VCH GmbH. This is an open access article under the terms of the Creative Commons Attribution License, which permits use, distribution and reproduction in any medium, provided the original work is properly cited.

DOI: 10.1002/adom.202002039 devices during the past decade, ${ }^{[5-7]}$ the performance of doped electron-transport materials is still limited. ${ }^{[8-10]}$ One key criterion for selecting $\mathrm{n}$-dopants is their ionization energy (IE), which should be lower than the electron affinity (EA) of the semiconducting host to facilitate integer charge transfer. Consequently, many n-dopants exhibit a high susceptibility to oxidation and are unstable in air. ${ }^{[8,11-14]}$ Alkali metals can be employed as strong reducing agents, but these small atoms tend to diffuse throughout the organic layer, resulting in device instabilities. ${ }^{[9,15]}$ Therefore, molecular dopants were introduced, which have a lower tendency to diffuse due to their larger size. Among the materials available for organic semiconductor $n$-doping, such as tetrathianaphthacene (TTN) and the radical obtained on heating 2-(2-methoxyphenyl)-1,3-dimeth-yl-1H-benzoimidazol-3-ium chloride (o-MeO-DMBI-Cl), ${ }^{[16,17]}$ metal-organic compounds like bis(terpyridine)ruthenium(II) ([Ru(terpy $\left.\left.)_{2}\right]^{0}\right)$, and organometallic sandwich compounds like cobaltocene $\left(\mathrm{CoCp}_{2}\right)$ and decamethylcobaltocene $\left(\mathrm{CoCp}_{2}{ }_{2}\right)$, having a low IE, have proven to act as efficient electron donors. ${ }^{[18-20]}$ However, instability of the neutral state in air and high vapor pressure complicate the handling of these materials, making them problematic from an application point of view. This issue was partially circumvented by utilizing organometallic dimers, e.g., (pentamethyl-cyclopentadienyl)(cyclopentadienyl)rhodium dimer ([RhCp* $\left.{ }^{*}\right]_{2}$ ) and (pentamethylcyclopentadienyl) (1,3,5-trimethylbenzene)ruthenium dimer ([RuCp*Mes $]_{2}$ ). These dimers consist of two organometallic monomers, where a group 8 metal (e.g., ruthenium) or a group 9 metal (e.g., 
$\mathrm{P}(\mathrm{NDI} \text { 2OD-T })_{2}$

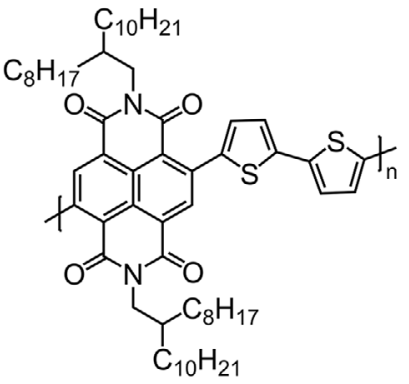

OPR

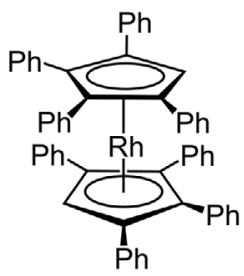

Figure 1. Chemical structures of $\mathrm{P}\left(\mathrm{NDI} / 2 \mathrm{OD}-\mathrm{T}_{2}\right)$ and the $\mathrm{n}$-dopant $\mathrm{OPR}$ $\left[\left(\mathrm{C}_{5} \mathrm{HPh}_{4}\right)_{2} \mathrm{Rh}\right]$.

rhodium) is sandwiched between a cyclopentadienyl and an arene group or two cyclopentadienyl groups, respectively. Their 18-electron configurations lead to moderate air stability. ${ }^{[12,21,22]}$ These compounds are well suited for n-type doping, since, for example, the air-stable $\left[\mathrm{RhCp}^{*} \mathrm{Cp}\right]_{2}$ dimer can dissociate into two monomers with a 19-electron configuration when heated, resulting in two highly reducing monomers. As the second key criterion for dopant selection, the abovementioned diffusion of molecular dopants should be minimized in order to improve device stability. ${ }^{[23,24]}$ Attaching bulky and inert side groups could help inhibit diffusion. This strategy also increases the distance between the ionized semiconductor segment and the dopant counterion, which is anticipated to result in decreased Coulomb interaction and thus improved charge carrier transport properties. ${ }^{[25,26]}$

Considering the two abovementioned dopant-selection criteria, we chose to investigate the $\mathrm{n}$-doping capability of the metallocene 1,2,3,4,1',2, $2^{\prime}, 4^{\prime}$-octaphenylrhodocene ${ }^{[27]}$ (OPR, $\left(\mathrm{C}_{5} \mathrm{HPh}_{4}\right)_{2} \mathrm{Rh}$, see Figure 1 for chemical structure). Like cobaltocene, OPR is a monomeric 19-electron sandwich compound, acting as a strong reducing agent (we find an oxidation potential of $\approx-1.5 \mathrm{~V}$ vs $\mathrm{FeCp}_{2}{ }^{+} / \mathrm{FeCp}_{2}$, measured on a salt of the corresponding cation, (OPR) $\mathrm{PF}_{6}$, in $\mathrm{CH}_{2} \mathrm{Cl}_{2}$ solution containing $0.1 \mathrm{M} \mathrm{NBu}_{4} \mathrm{PF}_{6}$ as supporting electrolyte with differential pulse voltammetry; this is similar to values previously reported in other solvents ${ }^{[27]}$ and corresponds to an IE of $\approx 3.3 \mathrm{eV}$. It undergoes a one-electron oxidation to obtain a cation in which the metal center has a stable 18-electron configuration. ${ }^{[12]}$ The eight peripheral phenyl groups render OPR significantly more bulky than $\mathrm{CoCp}_{2}$ or $\mathrm{CoCp}_{2}^{*}$. As organic semiconductor, we chose the widely used electron-transporting polymer poly $\left\{\left[N, N^{\prime}\right.\right.$ bis(2-octyldodecyl)naphthalene-1,4,5,8-bis(dicarboximide)-2,6diyl]-alt-5,5'-(2,2'-bithiophene)\} [P(NDI2OD-T 2$)], \quad$ chemical structure shown in Figure 1), which has a rather high EA of $3.95 \mathrm{eV}$ (corresponding to a reduction potential of $\approx-1.0 \mathrm{~V}$ vs $\mathrm{FeCp}_{2}{ }^{+} / \mathrm{FeCp}_{2}$, which we measure in $\mathrm{CH}_{2} \mathrm{Cl}_{2}$ solution containing $0.1 \mathrm{M} \mathrm{NBu}_{4} \mathrm{PF}_{6}$ as supporting electrolyte with differential pulse voltammetry, with similar values measured using a variety of techniques and media in a variety of other literature studies). ${ }^{[22,28,29]}$ For OPR-doped P(NDI2OD- $T_{2}$ ) films, we observe a bulk conductivity enhancement by three orders of magnitude, and the contact resistance to indium tin oxide (ITO) electrodes is decreased by about five orders of magnitude. The latter is due to an accumulation of dopants at the

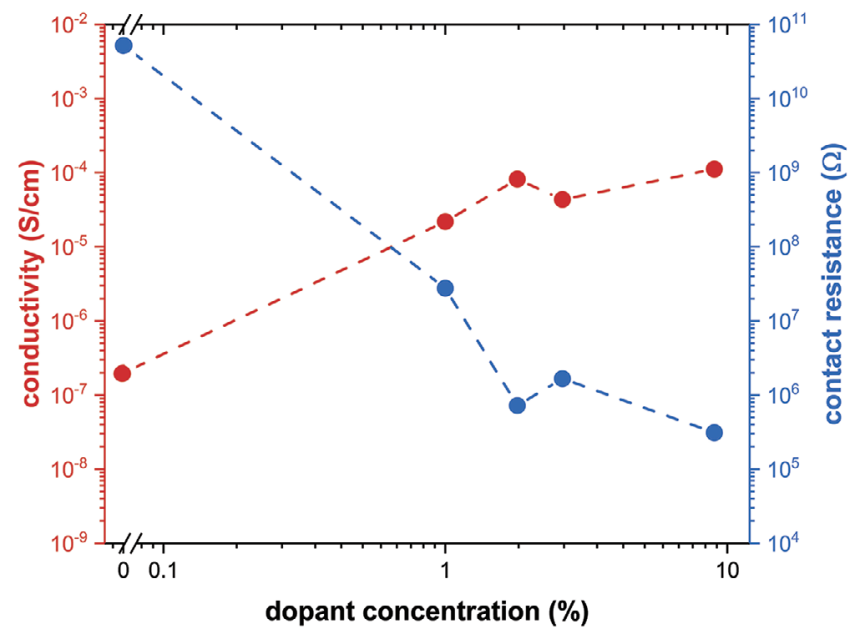

Figure 2. Conductivity and contact resistance of OPR-doped P(NDI2OD$T_{2}$ ) thin films as a function of dopant concentration measured under inert nitrogen atmosphere. The values for the undoped P(NDI2OD- $\left.T_{2}\right)$ film are taken from literature. ${ }^{30]}$

ITO interface, where electron transfer from OPR to the electrode lowers the work function and thus electron injection barrier into $\mathrm{P}\left(\mathrm{NDI} 2 \mathrm{OD}-\mathrm{T}_{2}\right)$. Optical absorption spectroscopy and electron paramagnetic resonance (EPR) reveal that the bulk conductivity increase has two contributions. Low-level doping increases the electron density in the polymer and trap filling enhances the charge carrier mobility.

\section{Results and Discussion}

The bulk conductivity and contact resistance of P(NDI2OD$\mathrm{T}_{2}$ ) films cast from chlorobenzene (CB) solutions of polymer and dopant measured in two-terminal devices as a function of dopant concentration are plotted in Figure 2. The conductivity increases by up to three orders of magnitude with increasing dopant concentration, reaching a value of $1.1 \times 10^{-4} \mathrm{~S} \mathrm{~cm}^{-1}$ at $9 \%$ dopant concentration. Since the conductance of the pristine $\mathrm{P}\left(\mathrm{NDI} 2 \mathrm{OD}-\mathrm{T}_{2}\right)$ film prepared from $\mathrm{CB}$ was too low to provide a reliable signal, a reference conductivity value of $2 \times 10^{-7} \mathrm{~S} \mathrm{~cm}^{-1}$ is taken from a previous $\mathrm{P}\left(\mathrm{NDI} 2 \mathrm{OD}-\mathrm{T}_{2}\right.$ ) study as upper limit. ${ }^{[30]}$ Although using the same method as in that study, a variation in conductance can be due to the use of different solvents and processing conditions, as these parameters affect the polymer morphology and thus conductivity. ${ }^{[31-33]}$ However, use of this reference value allows establishing an increase in conductivity by at least three orders of magnitude upon doping. The contact resistance to the ITO electrodes is reduced by about five orders of magnitude, from $5.2 \times 10^{10} \Omega$ (reference value taken from a previous study ${ }^{[30]}$ ) to $3.1 \times 10^{5} \Omega$ for the highest dopant concentration. It should be noted that the blip in conductivity and dip in contact resistance at $2 \%$ dopant concentration, respectively, has no significance. It is within the experimental error and appears overamplified due to the logarithmic scale. The values are similar within the measurement uncertainties. The current versus voltage and resistance versus channel length curves of the plane two-terminal P(NDI2OD- $\mathrm{T}_{2}$ ) devices for different 


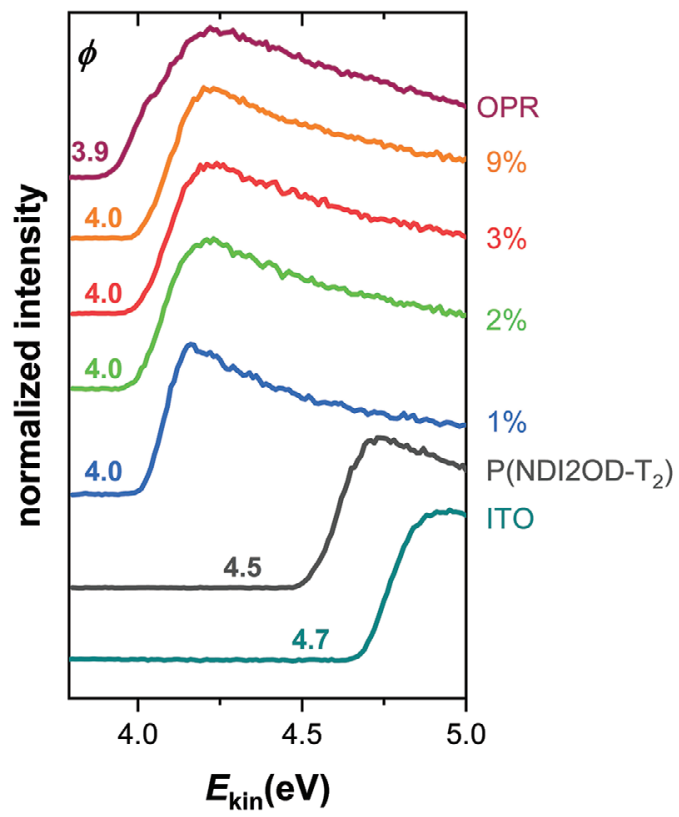

Figure 3. UPS SECO spectra of pristine OPR and $\mathrm{P}\left(\mathrm{NDI} 2 \mathrm{OD}-\mathrm{T}_{2}\right), \mathrm{ITO}$, and OPR-doped P(NDI2OD-T ${ }_{2}$ ) films. $\Phi$ stands for the work function. All spectra are normalized and vertically shifted for clarity.

dopant concentration are shown in Figure S1, Supporting Information. To unravel the causes of contact resistance decrease and bulk conductivity increase, we employed ultraviolet photoelectron spectroscopy (UPS), X-ray photoelectron spectroscopy (XPS), UV-vis-NIR absorption, and EPR measurements.

Photoelectron spectroscopy was used to clarify whether the decreased contact resistance is predominantly due to polymer/ electrode interface effects or due to efficient bulk doping of the polymer. At first, secondary electron cutoff (SECO) spectra of OPR-doped P(NDI2OD-T 2 ) films were measured, displayed in Figure 3 . The pristine $\mathrm{P}\left(\mathrm{NDI} 2 \mathrm{OD}-\mathrm{T}_{2}\right)$ film on ITO gives a work function of $4.5 \mathrm{eV}$, while the work function of bare ITO is $4.7 \mathrm{eV}$. Such work function reduction is in line with the expected push-back effect for conductive oxide surfaces. ${ }^{[34]}$ A film of $1 \%$ OPR-doped P(NDI2OD- $\mathrm{T}_{2}$ ) on ITO reduces the work function to $4.0 \mathrm{eV}$. Higher dopant concentrations do not lead to any further work function reduction because the Fermi level is apparently pinned at the $\mathrm{P}\left(\mathrm{NDI} 2 \mathrm{OD}-\mathrm{T}_{2}\right)$ conduction band $(\approx 3.95 \mathrm{eV})$ at $1 \%$ dopant concentration. ${ }^{[22,30,35]}$ The pronounced work function decrease of $0.5 \mathrm{eV}$ is unexpected for such low dopant concentration, and could indicate a preferential accumulation of OPR molecules at the ITO electrode. In this scenario, electron transfer from the dopants to ITO could occur, leading to a decrease of the electrode work function. ${ }^{[36-39]}$ This hypothesis is supported by the observation that deposition of OPR directly onto ITO (OPR film thickness of about $5 \mathrm{~nm}$ ) gives a work function of $3.9 \mathrm{eV}$, i.e., almost equal to that of doped P(NDI2OD$\mathrm{T}_{2}$ ) films. Furthermore, ITO coated with polyethylenimine (PEI) also induced Fermi level pinning at the P(NDI2OD- $\mathrm{T}_{2}$ ) conduction band, and the resulting sample work function was also $4.0 \mathrm{eV} .^{[30]}$ However, the polymer PEI is not a one-electron reductant as is OPR, and the reason for work function reduction was related to its intrinsic dipole moment instead of electron transfer. ${ }^{[40]}$

To confirm the accumulation of OPR at the interface to ITO, XPS measurements were performed for a $9 \%$ doped $\mathrm{P}\left(\mathrm{NDI} 2 \mathrm{OD}-\mathrm{T}_{2}\right)$ film, and after peeling this film from the ITO substrate with adhesive tape. While scans of the Rh $3 p$ core level region for the intact film did not indicate the presence of OPR near the polymer film surface, a clear Rh $3 p$ signal was detected after peeling (see Figure 4a). Sulfur and nitrogen core level signals were absent after peeling, showing that P(NDI2OD- $\mathrm{T}_{2}$ ) was effectively removed. These observations provide clear evidence a)
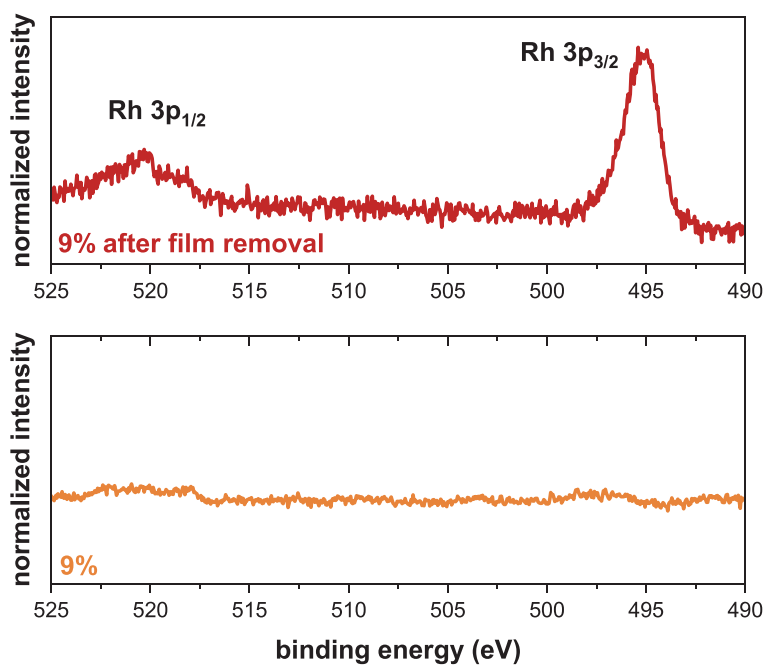

b)

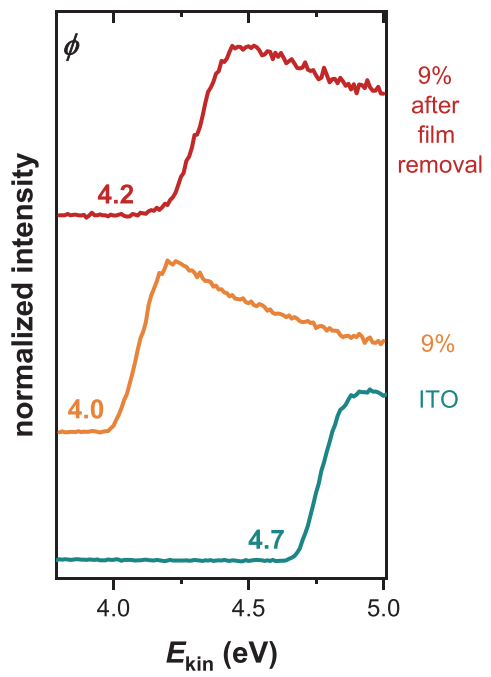

Figure 4. a) XPS detail scans of the Rh $3 p$ region of $9 \%$ OPR-doped P(NDI2OD- $T_{2}$ ) film before (bottom) and after (top) film removal. The spectra are normalized with respect to each other. b) UPS SECO spectra of ITO, $9 \%$ OPR-doped P(NDI2OD-T 2 ) film before and after the film removal procedure. $\Phi$ represents the work function. All spectra are normalized and vertically shifted for clarity. 

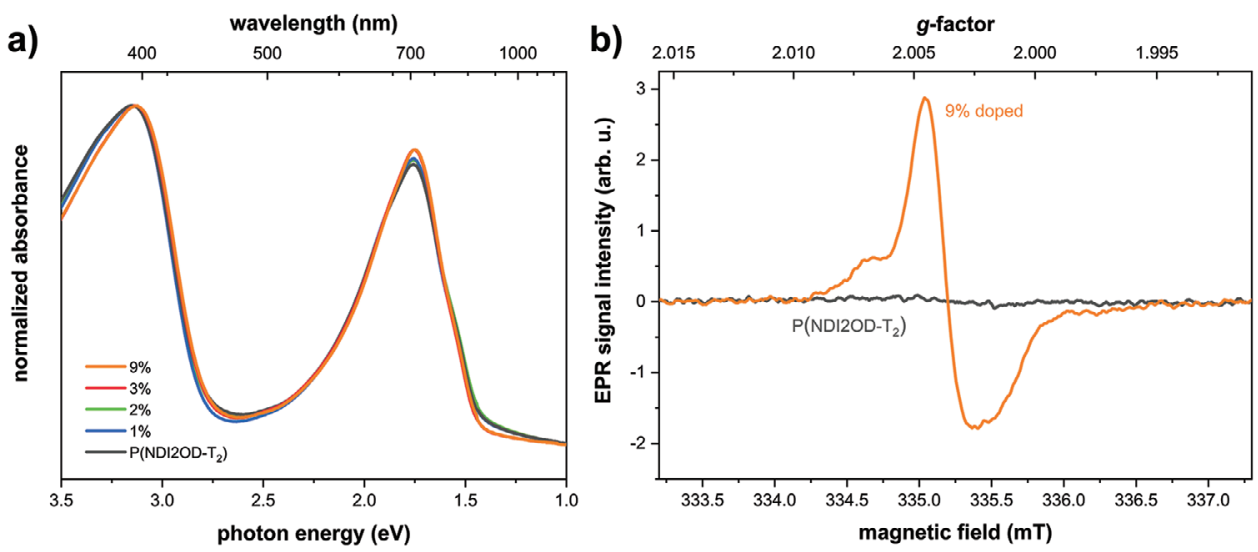

Figure 5. a) Optical absorption spectra of OPR-doped P(NDI2OD- $\left.\mathrm{T}_{2}\right)$. All spectra are normalized to their highest absorbance peak. b) X-band continuous wave EPR spectra recorded for undoped and 9\% OPR-doped P(NDI2OD-T $)$ films at room temperature.

for the preferential accumulation of OPR at ITO, and a very low dopant concentration near the polymer surface, possibly even in the bulk. Additionally, the sample work function increased just slightly after peeling (by $0.2 \mathrm{eV}$, see Figure $4 \mathrm{~b}$ ). This suggests that the contact resistance decrease is indeed rather due to Fermi level pinning, and thus a minimized electron injection barrier, rather than high-level polymer bulk doping.

Next, we examine the reasons underlying the observed bulk conductivity increase of OPR-doped P(NDI2OD-T 2 ). Optical absorption spectroscopy was performed because characteristic absorption features of polarons, being the charged state of a polymer segment, would be a direct manifestation of doping. The spectra in Figure 5a show the typical absorption of $\mathrm{P}\left(\mathrm{NDI} 2 \mathrm{OD}-\mathrm{T}_{2}\right)$ films, in agreement with literature. ${ }^{[1,42]}$ The peak at $1.8 \mathrm{eV}$ is associated with a transition with bithiophene to naphthalene bis(dicarboximide) charge-transfer character; the peak at $3.1 \mathrm{eV}$ is due to a localized transition associated with the acceptor moiety. ${ }^{[2,43]}$ OPR exhibits only weak absorption in the energy range shown here. Upon addition of OPR to P(NDI2OD-T 2 ), no additional absorption features appear and the spectra up to $9 \%$ dopant concentration are virtually identical. Since the optical spectra of doped P(NDI2OD- $\mathrm{T}_{2}$ ) show additional polaron peaks at $\approx 2.6$ and $1.6 \mathrm{eV},{ }^{[41,42]}$ the density of polarons induced by doping with up to $9 \%$ OPR under the present conditions is below the detection limit of standard absorption spectroscopy.

EPR measurements were thus performed, which enable the detection of unpaired spins with higher sensitivity. The first-derivative continuous wave EPR spectra of undoped and $9 \%$ doped $\mathrm{P}\left(\mathrm{NDI} 2 \mathrm{OD}-\mathrm{T}_{2}\right)$ films are depicted in Figure $5 \mathrm{~b}$. The pristine polymer film exhibits a very faint signal. The doped P(NDI2OD- $\mathrm{T}_{2}$ ) film gives a notable EPR signal at a g-value of about $2.0035 \pm 0.0005$, which is in agreement with literature values for the radical anions of P(NDI2OD- $\mathrm{T}_{2}$ ) and the corresponding monomer obtained upon doping with tetrakis(dimethylamino)ethylene (TDAE). ${ }^{[44,45]}$ No contributions to the EPR spectrum attributable to dopant species were detected in the room-temperature EPR measurements. This is consistent with expectations for both the OPR cation, which is a closed-shell 18-electron species, and neutral OPR, which, along with other 19-electron metallocenes, is not detectable at room temperature due to fast relaxation. ${ }^{[27]}$ The observed signal is thus attributed exclusively to radical anions on the polymer backbone and spin quantification gives a spin density of $\leq(1.2 \pm 0.5) \times 10^{16} \mathrm{~cm}^{-3}\left(\approx 1.8 \times 10^{-5}\right.$ spins per polymer repeating unit) and about $(9.7 \pm 2.6) \times 10^{16} \mathrm{~cm}^{-3}\left(\approx 1.4 \times 10^{-4}\right.$ spins per polymer repeating unit) for the undoped and doped films, respectively. The spin density in 9\% OPR-doped P(NDI2OD- $\mathrm{T}_{2}$ ) films is thus increased by only one order of magnitude, indicating very ineffective doping because the nominal dopant concentration in solution was almost $10^{-1}$ per polymer repeat unit. The small increase in charge-carrier density is in line with an OPR level in the polymer film sufficiently low as to be undetectable by XPS. This is in line with the finding from XPS discussed above that OPR accumulates at the ITO interface.

As a side note, the observed structure of the EPR spectrum of the doped P(NDI2OD- $T_{2}$ ) film is likely a result of g-anisotropy as well as anisotropic hyperfine interactions between the unpaired electron spin and the nitrogen and hydrogen nuclei in $\mathrm{P}\left(\mathrm{NDI} 2 \mathrm{OD}-\mathrm{T}_{2}\right)$. The line shape may additionally be affected by partial ordering in the films. The observation of a clear asymmetry in the EPR spectra of the OPR-doped P(NDI2OD- $T_{2}$ ) films investigated in this study, in contrast to previous measurements on PEI-doped P(NDI2OD- $\left.T_{2}\right)$ films prepared from chloroform that showed symmetric EPR signals, ${ }^{[30]}$ could potentially be due to averaging of the anisotropic interactions due to fast hopping and/or exchange in the previously studied films with a significantly higher spin density or to differences in molecular ordering. An in-depth analysis of the EPR results is, however, beyond the scope of the present work.

Having established that OPR accumulates at ITO and significantly reduces the electron injection barrier and having detected only low-level doping of P(NDI2OD- $\mathrm{T}_{2}$ ) with OPR by EPR, the observed increase in conductivity by three orders of magnitude remains to be explained. Since we did not observe apparent morphology differences between doped and undoped polymer films, significant differences in molecular packing and thus carrier mobility seem unlikely. In organic semiconductors, however, the carrier mobility can be significantly reduced by gap states that act as traps for charge carriers. Besides trap states that originate from impurities and static or dynamic disorder in the semiconductor, ${ }^{[46-48]}$ oxygen- and water-related traps were also found to limit electron transport. ${ }^{[4]}$ Since 
the EA of P(NDI2OD-T $)_{2}$ is higher than that of oxygen- and water-related traps in films, hydrated oxygen complexes can be excluded as possible causes for trap states. The increase of charge carrier density due to doping leads to trap-filling, rendering them electronically inactive. This compares well with previous observations. Higgins et al. have shown that existing trap states in undoped P(NDI2OD- $\mathrm{T}_{2}$ ) can be rendered electronically inert by ultralow $n$-doping of the polymer with the dimer $\left[\mathrm{RhCp}{ }^{*} \mathrm{Cp}\right]_{2}$, resulting in an increased electron mobility by two orders of magnitude. ${ }^{[31]}$ Similarly, Olthof et al. have shown that the addition of $\left[\mathrm{RuCp}{ }^{*} \mathrm{Mes}\right]_{2}$ to the fullerene $\mathrm{C}_{60}$ leads to filling of trap states, increasing the mobility by nearly three orders of magnitude. ${ }^{[50]}$

As a further check, Fermi-Dirac statistics and Gaussian density of states were used in order to estimate the dependence of the Fermi level shift on the charge carrier density. ${ }^{[51]}$ As can be seen from Figure S2, Supporting Information, a Fermi level shift by $0.5 \mathrm{eV}$ (as determined for all OPR-doped films from the work function behavior, see Figure 3) requires an increase in electron density of almost six orders of magnitude. Since the film conductivity was enhanced by only up to three orders of magnitude and EPR gave only one order of magnitude carrier density increase, the decrease in work function cannot be assigned to doping of $\mathrm{P}\left(\mathrm{NDI} 2 \mathrm{OD}-\mathrm{T}_{2}\right)$ itself but must be due to the OPR-induced work function reduction of ITO. The induced Fermi level shift of several hundred meV by a dipole at the semiconductor/ITO interface is also supported by considering the following. Assuming electrostatic potential changes perpendicular to the interface plane, the simplest case of an unperturbed interface dipole can be described via the Helmholtz equation $^{[52]}$

$\Delta \phi=\frac{e \cdot n \cdot p_{\perp}}{\varepsilon_{0} \cdot \varepsilon_{\mathrm{r}}}$

where $n$ is the number of transferred charges per unit area, $p_{\perp}$ is the dipole magnitude perpendicular to the plane, $e$ the elementary charge, and $\varepsilon_{0}$ and $\varepsilon_{\mathrm{r}}$ the vacuum and relative dielectric permittivity. Assuming reasonable values of 0.2 molecules $\mathrm{nm}^{-2}$, a molecule-metal bonding distance of $0.5 \mathrm{~nm}$ to obtain $p_{\perp}$, and a typical dielectric constant value for organic semiconductors of 3 , yields a work function change of $0.6 \mathrm{eV}$. Therefore, the work function reduction can indeed be assigned to interface dipole formation due to OPR-ITO charge transfer.

The remaining question is why $\mathrm{P}\left(\mathrm{NDI} 2 \mathrm{OD}-\mathrm{T}_{2}\right)$ is barely doped when films are cast from CB solution, despite the fact that the oxidation potential of OPR is at a significantly more cathodic potential than the reduction potential of $\mathrm{P}(\mathrm{NDI} 2 \mathrm{OD}$ $\mathrm{T}_{2}$ ). In fact, we found that notable doping of $\mathrm{P}\left(\mathrm{NDI} 2 \mathrm{OD}-\mathrm{T}_{2}\right)$ occurred when OPR, dissolved in tetrahydrofuran, was deposited by drop-casting onto a pristine polymer film (see Figure S3, Supporting Information). For this sequential doping, we observe the clear emergence of the two polaron absorption features at about 1.4 and $2.6 \mathrm{eV}$. Therefore, we propose that the solvation-shell of OPR and its $\approx 10$ times lower solubility compared to $\mathrm{P}\left(\mathrm{NDI} 2 \mathrm{OD}-\mathrm{T}_{2}\right)$ in $\mathrm{CB}$ cause the observed behavior. The solvation-shell and the bulky phenyl peripheral groups of the dopant result in a long distance between the OPR metal center and solvated polymer chains. This would effectively suppress charge transfer between the two components already in solution, which we indeed observe in experiment (see Figure S4, Supporting Information). During film formation, the solvent evaporates and OPR precipitates at the substrate before the polymer does, because of the different solubility, in particular the poor solubility of neutral OPR arising from its large size and its ability to engage in extensive van der Waals interactions with its neighbors. This mechanism is in line with the experimental observations and highlights the importance of adjusting many dopant properties simultaneously to achieve superior doping in thin films.

\section{Conclusions}

The n-type dopant OPR mixed with the electron conducting polymer semiconductor P(NDI2OD- $\mathrm{T}_{2}$ ) in CB solution undergoes negligible charge transfer in solution prior to film formation, as result of weak electronic coupling imposed by the solvation-shell and the dopant's bulky peripheral groups. During thin film formation, the lower solubility of the dopant compared to the polymer leads to accumulation of OPR at the ITO substrate. Here, electron transfer from OPR to ITO occurs, which lowers the electrode's work function by $0.5 \mathrm{eV}$ and minimizes the electron injection barrier into the polymer via Fermi level pinning. Residual OPR within the polymer bulk leads to low-level doping and increased film conductivity by up to one order of magnitude. A simultaneous further boost of conductivity by two orders of magnitude is ascribed to trap filling and concomitant charge carrier mobility increase. Importantly, the combination of methods employed here to derive a coherent understanding of the phenomena occurring in OPR-doped P(NDI2OD- $\left.\mathrm{T}_{2}\right)$ films demonstrates that the observation of a Fermi level shift in photoemission spectroscopy is not sufficient to conclude on effective doping. Rather, preferential dopant migration towards the conductive substrate and alteration of its work function can mimic the effects expected for doping. We note that these phenomena could have implications for improving contacts between active layers and electron collecting electrodes in some systems.

\section{Experimental Section}

P(NDI2OD-T 2 ) was purchased from Ossila Ltd., and used without further purification. The metallocene OPR was synthesized according to the procedure described. ${ }^{[27]}$ Stock solutions with concentrations of 0.5 and $5 \mathrm{mg} \mathrm{mL}^{-1}$ for OPR and $\mathrm{P}\left(\mathrm{NDI} 2 \mathrm{OD}-\mathrm{T}_{2}\right)$, respectively, were prepared under nitrogen atmosphere in an inert gas box $(<0.1 \mathrm{ppm}$ $\mathrm{H}_{2} \mathrm{O},<0.1 \mathrm{ppm} \mathrm{O}_{2}$ ) using dried and degassed $\mathrm{CB}$. To enable complete dissolution of the materials, the stock solutions were stirred overnight. Thin films were prepared via spin-coating using standard laboratory spin-coaters at $1000 \mathrm{rpm}$ for $1 \mathrm{~min}$ to achieve the desired thickness. The reported dopant concentration, given in $\%$, is $c=N_{D} /\left(N_{D}+N_{P}\right)$, where $N_{D}$ is the number of dopant molecules and $N_{P}$ is the number of monomer units of the polymer as contained in the solutions.

Optical absorption spectroscopy was performed using a Lambda 950 UV-vis-NIR spectrophotometer (PerkinElmer, Inc.). Doped thin films were prepared on quartz glass substrates with thickness between 20 and $60 \mathrm{~nm}$. Oxygen exclusion during the optical measurements was ensured by using nitrogen-filled boxes with two quartz glass windows, sealed by using a vinyl gasket, in which the samples were mounted. 
Conductivity measurements were performed under nitrogen atmosphere using pre-patterned glass substrates with an interdigitated ITO electrode structure, purchased from Ossila Ltd. These substrates consist of five individual electrode pairs, each having $100 \mathrm{~nm}$ thick electrodes with five channels of $6 \mathrm{~mm}$ width and channel lengths ranging from 50 to $200 \mu \mathrm{m}$. This enables the separation of contact and channel resistance, using the transfer length method. ${ }^{[53]}$ Current-voltage $(I-V)$ measurements were conducted using an $I-V$ test board from Ossila and a Keithley 2635A source meter. The contact resistance $R_{\text {contact }}$ was determined as the intersection of the linearly fitted resistancechannel length $(R-L)$ curve with the $y$-axis. Using the inverse slope $S$ of the $R-L$ curve, the film thickness $d$ and the electrode width $w$, the actual bulk conductivity (excluding influences of the contact resistance) can be determined via $\sigma=\frac{S}{w \cdot d}$, as described elsewhere. ${ }^{[30]}$ The sample conductivity was determined at low electric fields from the Ohmic regime of the $I-V$ scans.

For EPR measurements, $50 \mu \mathrm{L}$ of undoped and 9\% OPR-doped $\mathrm{P}\left(\mathrm{NDI} 2 \mathrm{OD}-\mathrm{T}_{2}\right)$ solutions (prepared at a constant $\mathrm{P}\left(\mathrm{NDI} 2 \mathrm{OD}-\mathrm{T}_{2}\right.$ ) concentration), respectively, were filled into $3 \mathrm{~mm}$ ID quartz tubes inside a nitrogen-filled inert gas box. The solvent was evaporated under vacuum, resulting in a film on the inner walls of the EPR tube. The tubes were then backfilled with helium and flame-sealed. X-band continuous wave EPR measurements were performed at room temperature using an ER 4122 SHQE resonator on a laboratory-built spectrometer, which consists of a Bruker ER 041 MR microwave bridge with an ER $048 \mathrm{R}$ microwave controller and an AEG electromagnet with a Bruker $\mathrm{BH} 15$ Hall effect field controller. A Stanford Research SR810 lock-in amplifier in combination with a Wangine WPA-120 modulation amplifier was used for field modulation and lock-in detection. The spectra were acquired at a microwave frequency of $9.4 \mathrm{GHz}$ and a microwave power of $63 \mu \mathrm{W}$ with a $100 \mathrm{kHz}$ modulation frequency and $0.1 \mathrm{mT}$ modulation amplitude. The spectrum recorded for an empty EPR tube inside the resonator cavity was used to perform a background correction. The field was calibrated with a standard $\mathrm{N} @ \mathrm{C}_{60}$ sample with a known g-value. ${ }^{[54]}$ Spin quantification was performed with reference to a standard sample of TEMPOL in toluene with a known spin concentration, taking into account the quality factor of the resonator, determined from the mode picture for each measurement. ${ }^{[5]}$

UPS and XPS measurements of OPR-doped P(NDI2OD- $\left.\mathrm{T}_{2}\right)$ thin films on ITO substrates were performed in an ultrahigh vacuum chamber with a base pressure of $10^{-9}$ mbar. UPS was performed using a nonmonochromated helium-gas-discharge lamp $(21.22 \mathrm{eV})$. In order to avoid radiation damage of the samples, an $800 \mathrm{~nm}$ aluminum filter was used to attenuate the photon flux. For XPS measurements, nonmonochromated $\mathrm{Al} \mathrm{K} \alpha(1486.7 \mathrm{eV})$ radiation was used for excitation. The spectra were collected in normal emission using a SPECS Phoibos 100 hemispherical electron energy analyzer. A pass energy of $5 \mathrm{eV}$ was used for UPS measurements and $20 \mathrm{eV}$ for XPS. The SECO spectra were measured with a bias of $-10 \mathrm{~V}$ applied to the sample to clear the analyzer work function.

\section{Supporting Information}

Supporting Information is available from the Wiley Online Library or from the author.

\section{Acknowledgements}

This work was supported by the Deutsche Forschungsgemeinschaft (DFG) - project numbers 239543752 and 182087777-SFB 951, and the US National Science Foundation (DMR-1807797, the DMREF program DMR1729737). The authors thank Khaled Al Kurdi for running differential pulse voltammograms to check the electrochemical properties of OPR and $\mathrm{P}\left(\mathrm{NDI} 2 \mathrm{OD}-\mathrm{T}_{2}\right)$ under comparable conditions.

Open access funding enabled and organized by Projekt DEAL.

\section{Conflict of Interest}

The authors declare no conflict of interest.

\section{Data Availability Statement}

The data that support the findings of this study are available from the corresponding author upon reasonable request.

\section{Keywords}

conjugated polymers, doping, molecular electron donor, organic semiconductors

Received: November 26, 2020

Revised: December 15, 2020

Published online: February 2, 2021

[1] Z. Bin, Z. Liu, Y. Qiu, L. Duan, Adv. Opt. Mater. 2018, 6, 1800536

[2] S. Reineke, F. Lindner, G. Schwartz, N. Seidler, K. Walzer, B. Lüssem, K. Leo, Nature 2009, 459, 234.

[3] F. Selzer, C. Falkenberg, M. Hamburger, M. Baumgarten, K. Müllen, K. Leo, M. Riede, J. Appl. Phys. 2014, 115, 054515.

[4] S. Olthof, S. Singh, S. K. Mohapatra, S. Barlow, S. R. Marder, B. Kippelen, A. Kahn, Appl. Phys. Lett. 2012, 101, 253303.

[5] Y. Xu, H. Sun, A. Liu, H.-H. Zhu, W. Li, Y.-F. Lin, Y.-Y. Noh, Adv. Mater. 2018, 30, 1801830.

[6] B. Lüssem, C.-M. Keum, D. Kasemann, B. Naab, Z. Bao, K. Leo, Chem. Rev. 2016, 116, 13714

[7] B. Lüssem, M. Riede, K. Leo, Phys. Status Solidi A 2013, 210, 9.

[8] P. Wei, J. H. Oh, G. Dong, Z. Bao, J. Am. Chem. Soc. 2010, 132, 8852.

[9] G. Parthasarathy, C. Shen, A. Kahn, S. R. Forrest, J. Appl. Phys. 2001, 89, 4986.

[10] P. Wei, T. Menke, B. D. Naab, K. Leo, M. Riede, Z. Bao, J. Am. Chem. Soc. 2012, 134, 3999.

[11] M. C. Gwinner, Y. Vaynzof, K. K. Banger, P. K. H. Ho, R. H. Friend, H. Sirringhaus, Adv. Funct. Mater. 2010, 20, 3457.

[12] S. Guo, S. B. Kim, S. K. Mohapatra, Y. Qi, T. Sajoto, A. Kahn, S. R. Marder, S. Barlow, Adv. Mater. 2012, 24, 699.

[13] C. K. Chan, E.-G. Kim, J.-L. Brédas, A. Kahn, Adv. Funct. Mater. 2006, $16,831$.

[14] M. Schwarze, M. L. Tietze, F. Ortmann, H. Kleemann, K. Leo, ACS Appl. Mater. Interfaces 2020, 12, 40566.

[15] R. Q. Png, M. C. Y. Ang, M. H. Teo, K. K. Choo, C. G. Tang, D. Belaineh, L. L. Chua, P. K. H. Ho, Nat. Commun. 2016, 7, 11948.

[16] S. Tanaka, K. Kanai, E. Kawabe, T. Iwahashi, T. Nishi, Y. Ouchi, K. Seki, Jpn. J. Appl. Phys. 2005, 44, 3760.

[17] M. Schwarze, B. D. Naab, M. L. Tietze, R. Scholz, P. Pahner, F. Bussolotti, S. Kera, D. Kasemann, Z. Bao, K. Leo, ACS Appl. Mater. Interfaces 2018, 10, 1340.

[18] K. Harada, A. G. Werner, M. Pfeiffer, C. J. Bloom, C. M. Elliott, K. Leo, Phys. Rev. Lett. 2005, 94, 036601.

[19] C. K. Chan, F. Amy, Q. Zhang, S. Barlow, S. Marder, A. Kahn, Chem. Phys. Lett. 2006, 431, 67.

[20] C. K. Chan, A. Kahn, Appl. Phys. A 2009, 95, 7.

[21] F. Zhang, C. Klein, E. Longhi, S. Barlow, S. R. Marder, G. Sarusi, A. Kahn, Chem. Mater. 2019, 31, 6624.

[22] Y. Qi, S. K. Mohapatra, S. Bok Kim, S. Barlow, S. R. Marder, A. Kahn, Appl. Phys. Lett. 2012, 100, 083305. 
[23] S. Duhm, I. Salzmann, B. Bröker, H. Glowatzki, R. L. Johnson, N. Koch, Appl. Phys. Lett. 2009, 95, 093305.

[24] S. Olthof, W. Tress, R. Meerheim, B. Lüssem, K. Leo, J. Appl. Phys. 2009, 106, 103711

[25] I. Salzmann, G. Heimel, J. Electron Spectros. Relat. Phenomena 2015, 204, 208.

[26] T. J. Aubry, K. J. Winchell, C. Z. Salamat, V. M. Basile, J. R. Lindemuth, J. M. Stauber, J. C. Axtell, R. M. Kubena, M. D. Phan, M. J. Bird, A. M. Spokoyny, S. H. Tolbert, B. J. Schwartz, Adv. Funct. Mater. 2020, 30, 2001800.

[27] J. E. Collins, M. P. Castellani, A. L. Rheingold, E. J. Miller, W. E. Geiger, A. L. Rieger, P. H. Rieger, Organometallics 1995, 14, 1232.

[28] J. Liu, L. Qiu, R. Alessandri, X. Qiu, G. Portale, J. Dong, W. Talsma, G. Ye, A. A. Sengrian, P. C. T. Souza, M. A. Loi, R. C. Chiechi, S. J. Marrink, J. C. Hummelen, L. J. A. Koster, Adv. Mater. 2018, 30, 1704630.

[29] X. Guo, M. D. Watson, Org. Lett. 2008, 10, 5333.

[30] K. F. Seidel, D. Lungwitz, A. Opitz, T. Krüger, J. Behrends, S. R. Marder, N. Koch, ACS Appl. Mater. Interfaces 2020, 12, 28801.

[31] A. Higgins, S. K. Mohapatra, S. Barlow, S. R. Marder, A. Kahn, Appl. Phys. Lett. 2015, 106, 163301.

[32] A. Luzio, L. Criante, V. D'Innocenzo, M. Caironi, Sci. Rep. 2013, 3, 3425.

[33] R. Steyrleuthner, R. Di Pietro, B. A. Collins, F. Polzer, S. Himmelberger, M. Schubert, Z. Chen, S. Zhang, A. Salleo, H. Ade, A. Facchetti, D. Neher, J. Am. Chem. Soc. 2014, 136, 4245.

[34] M. T. Greiner, M. G. Helander, W. M. Tang, Z. Bin Wang, J. Qiu, Z. H. Lu, Nat. Mater. 2012, 11, 76.

[35] J. Frisch, A. Vollmer, N. Koch, J. Appl. Phys. 2012, 112, 033712.

[36] K. Akaike, M. V. Nardi, M. Oehzelt, J. Frisch, A. Opitz, C. Christodoulou, G. Ligorio, P. Beyer, M. Timpel, I. Pis, F. Bondino, K. Moudgil, S. Barlow, S. R. Marder, N. Koch, Adv. Funct. Mater. 2016, 26, 2493.

[37] B. Bröker, R. P. Blum, J. Frisch, A. Vollmer, O. T. Hofmann, R. Rieger, K. Müllen, J. P. Rabe, E. Zojer, N. Koch, Appl. Phys. Lett. 2008, 93, 243303.

[38] W. E. Ford, D. Gao, N. Knorr, R. Wirtz, F. Scholz, Z. Karipidou, K. Ogasawara, S. Rosselli, V. Rodin, G. Nelles, F. von Wrochem, ACS Nano 2014, 8, 9173.
[39] G. Heimel, L. Romaner, E. Zojer, J.-L. Bredas, Acc. Chem. Res. 2008, 41, 721.

[40] Y. Zhou, C. Fuentes-Hernandez, J. Shim, J. Meyer, A. J. Giordano, H. Li, P. Winget, T. Papadopoulos, H. Cheun, J. Kim, M. Fenoll, A. Dindar, W. Haske, E. Najafabadi, T. M. Khan, H. Sojoudi, S. Barlow, S. Graham, J. L. Brédas, S. R. Marder, A. Kahn, B. Kippelen, Science 2012, 336, 327.

[41] D. Trefz, A. Ruff, R. Tkachov, M. Wieland, M. Goll, A. Kiriy, S. Ludwigs, J. Phys. Chem. C 2015, 119, 22760.

[42] S. Wang, H. Sun, U. Ail, M. Vagin, P. O. Å. Persson, J. W. Andreasen, W. Thiel, M. Berggren, X. Crispin, D. Fazzi, S. Fabiano, Adv. Mater. 2016, 28, 10764

[43] Z. Chen, Y. Zheng, H. Yan, A. Facchetti, J. Am. Chem. Soc. 2009, $131,8$.

[44] S. Wang, H. Sun, T. Erdmann, G. Wang, D. Fazzi, U. Lappan, Y. Puttisong, Z. Chen, M. Berggren, X. Crispin, A. Kiriy, B. Voit, T. J. Marks, S. Fabiano, A. Facchetti, Adv. Mater. 2018, 30, 1801898.

[45] S. Wang, D. Fazzi, Y. Puttisong, M. J. Jafari, Z. Chen, T. Ederth, J. W. Andreasen, W. M. Chen, A. Facchetti, S. Fabiano, Chem. Mater. 2019, 31, 3395.

[46] C. Krellner, S. Haas, C. Goldmann, K. P. Pernstich, D. J. Gundlach, B. Batlogg, Phys. Rev. B 2007, 75, 245115.

[47] W. L. Kalb, S. Haas, C. Krellner, T. Mathis, B. Batlogg, Phys. Rev. B 2010, 81, 155315.

[48] I. I. Fishchuk, A. Kadashchuk, S. T. Hoffmann, S. Athanasopoulos, J. Genoe, H. Bässler, A. Köhler, Phys. Rev. B 2013, 88, 125202.

[49] H. T. Nicolai, M. Kuik, G. A. H. Wetzelaer, B. De Boer, C. Campbell, C. Risko, J. L. Brédas, P. W. M. Blom, Nat. Mater. 2012, 11, 882.

[50] S. Olthof, S. Mehraeen, S. K. Mohapatra, S. Barlow, V. Coropceanu, J. L. Brédas, S. R. Marder, A. Kahn, Phys. Rev. Lett. 2012, 109, 176601.

[51] G. Paasch, S. Scheinert, J. Appl. Phys. 2010, 107, 104501.

[52] N. Koch, ChemPhysChem 2007, 8, 1438.

[53] S. Luan, G. W. Neudeck, J. Appl. Phys. 1992, 72, 766.

[54] J. J. Wittmann, T. V. Can, M. Eckardt, W. Harneit, R. G. Griffin, B. Corzilius, J. Magn. Reson. 2018, 290, 12.

[55] G. R. Eaton, S. S. Eaton, D. P. Barr, R. T. Weber, Quantitative EPR, Springer, Vienna 2010. 